\title{
RETRACTION
}

\section{Calmodulin kinase determines calcium-dependent facilitation of L-type calcium channels}

\section{Igor Dzhura, Yuejin Wu, Roger J. Colbran, Jeffrey R. Balser and Mark E. Anderson}

Nat. Cell Biol. 2, 173-177 (2000); published online 10 February 2000; retracted online 27 March 2015

We regret the lack of integrity of exemplar data in Figures 2-4 in this publication. The irregularities have been investigated by the co-authors and Vanderbilt University in collaboration with the US PHS Office of Research Integrity, and the investigation concluded that exemplar data in this publication were fabricated (http://ori.hhs.gov/content/case-summary-dzhura-igor). We have therefore decided to retract the publication and have been unable to contact the first author, Igor Dzhura, to sign the retraction statement.

The science showing CaMKII activates $\mathrm{CaV} 1.2 \mathrm{Ca}^{2+}$ channels and facilitates CaV1.2 current is now extensively validated, so our view is that the overall conclusions from this paper are valid. We apologize to the scientific community for any inconveniences or challenges resulting from the publication and retraction of this manuscript. 